\title{
Structural, dielectric, and electrical properties of lithium niobate microfibers
}

\author{
Cícero Rafael CENA ${ }^{a, *}$, Ajay Kumar BEHERA ${ }^{b}$, Banarji BEHERA ${ }^{b}$ \\ ${ }^{a}$ UFMS - Federal University of Mato Grosso do Sul, CP. 549, 79070-900, Campo Grande-MS, Brazil \\ ${ }^{b}$ School of Physics, Sambalpur University, JyotiVihar, Burla-768019, Odisha, India
}

Received: September 02, 2015; Revised: November 25, 2015; Accepted: December 03, 2015

(C) The Author(s) 2016. This article is published with open access at Springerlink.com

\begin{abstract}
Lithium niobate $\left(\mathrm{LiNbO}_{3}\right)$ fibers, obtained from the heat treatment of composite fibers (polymer/inorganic precursors), were successfully prepared by using the blow-spinning technique. A chemical solution containing $\mathrm{Li}$ and $\mathrm{Nb}$ ions, added to poly(vinyl pyrrolidone) solution, was used as precursor solution. The best condition for producing composite fibers was determined. The morphology of green and crystallized fibers was characterized by scanning electron microscopy (SEM) and revealed fibrous structure with an average diameter around $800 \mathrm{~nm}$. X-ray diffraction (XRD) measurement revealed a pure $\mathrm{LiNbO}_{3}(\mathrm{LN})$ phase formation. Detailed studies of dielectric response at various frequencies and temperatures exhibited a dielectric anomaly at $364{ }^{\circ} \mathrm{C}$. The electrical properties (impedance, modulus, and conductivity) of the fibers were studied using impedance spectroscopy technique. The contributions of grain and grain boundary effects were observed in the LN fibers. The activation energy of the composite fibers was found to be $1.5 \mathrm{eV}$ in the high temperature region $\left(325-400{ }^{\circ} \mathrm{C}\right)$.
\end{abstract}

Keywords: ceramics fibers; composites; chemical synthesis; impedance spectroscopy; dielectric properties

\section{Introduction}

In the past few decades, the processing of materials in the nanometer and micrometer scales has attracted numerous research groups. This can be attributed not only to being a relatively new area, but also, to the properties associated with different morphology and sizes from that observed at macroscale and its possible new applications [1]. In this context, different geometries may lead to various new properties and applications. The interest in nanostructured ceramics of the fibrous type is mainly due to the great potential for

* Corresponding author.

E-mail: cena@ifsp.edu.br applications and phenomenological studies because of its geometry [2].

The blow-spinning technique was first reported by Medeiros et al. [3] as an alternative to the well-established electrospinning technique [4] for the production of polymeric fibers. This new technique has an advantage of higher rate of fiber production, low production cost, and easy implementation. This is due to the application of high electrical field which is no longer necessary and open for the possibility of deposition of fibers on any type of target [3,5]. The blow-spinning technique basically consists of two concentric needles, in which, in the external canal, the air or other gas (at high pressure) is ejected, while in the internal channel, a polymer solution is injected 
simultaneously. The polymer solution is injected at a constant rate by a needle (internal channel). One drop of solution is formed at the tip of the needle. By the action of drag force, originating from the pressure difference caused by the flow of air, it is stretched to give rise to the fiber jet. The jet ejected from the needle tip is accelerated which leaves the polymer fibers $[3,5]$.

Poly(vinyl pyrrolidone) (PVP) has been extensively used in the production of fibers via electrospinning technique [6-9]. A systematic study was carried out to obtain PVP fibers by blow-spinning technique and evaluated the samples in terms of their morphology by parameters such as the diameter size distribution, homogeneity, and shape of fibers. Ferroelectric fibers, such as barium titanate $\left(\mathrm{BaTiO}_{3}\right)$ [6], lead zirconium titanate (PZT) [7], and barium strontium titanate (BST) [8], have been successfully produced as nanofibers. To the best of our knowledge, there is no previous report on the fabrication of lithium niobate nanofibers. Lithium niobate (LN) is a perovskite ferroelectric phase with an $\mathrm{ABO}_{3}$ type crystal structure, and its properties have great interest for potential applications in optical, nonlinear optical, and optoelectronic technologies $[10,11]$. Despite various investigations about this material and its potential applications, a relatively new synthesis route and its properties are discussed.

For the first time, this paper reports on the fabrication of lithium niobate microfibers by blow-spinning using poly(vinyl pyrrolidone) as precursor solution that contains $\mathrm{Li}$ and $\mathrm{Nb}$ ions. This work was carried out to study the effect of fiber morphology on phase formation and dielectric and electrical properties of the microfibers.

\section{Experimental}

Poly(vinyl pyrrolidone) (PVP, $\left.\left(\mathrm{C}_{6} \mathrm{H}_{9} \mathrm{NO}\right)_{n}\right)$ with average molecular weight of 360.000 was supplied in powder form from M/s. Sigma-Aldrich Chemical Company. The PVP solvent was a mixture of isopropyl alcohol $\left(\mathrm{AI}\left(\mathrm{C}_{3} \mathrm{H}_{8} \mathrm{O}\right)\right)$ and deionized water in different volume proportions. The polymer concentration was kept at $0.10 \mathrm{~g} / \mathrm{mL}$. The rheological characterization of the polymeric solution was performed by a rotational rheometer from Brookfield DV-II Pro model with coaxial-cylinder geometry. The $\mathrm{LiNbO}_{3}$ (LN) precursor solution was prepared by dissolving lithium carbonate $\left(\mathrm{Li}_{2} \mathrm{CO}_{3}, \mathrm{M} / \mathrm{s}\right.$. Synth Chemical Company) and ammonium niobium oxalate $\left(\mathrm{NH}_{4}\left[\mathrm{NbO}\left(\mathrm{C}_{2} \mathrm{O}_{4}\right)_{2}\right.\right.$ $\left.\left(\mathrm{H}_{2} \mathrm{O}\right)\right]\left(\mathrm{H}_{2} \mathrm{O}\right)_{n}$, CBMM Company, containing $27.6 \%$ of
$\left.\mathrm{Nb}_{2} \mathrm{O}_{5}(w / w)\right)$ in deionized water. Initially PVP polymeric fibers were produced by "blow-spinning" technique under different solvent proportions of $\mathrm{AI}$ and $\mathrm{H}_{2} \mathrm{O}$. The best condition for polymeric fiber formation was determined and kept preferentially for future composite fiber deposition. The standard processing condition included an injection rate of $0.11 \mathrm{~mL} / \mathrm{min}$, a gas pressure of $120 \mathrm{kPa}$, a work distance of $25 \mathrm{~cm}$, a distance of $5 \mathrm{~mm}$ that the needle protruded beyond the nozzle base, and a needle diameter of $\sim 0.424 \mathrm{~mm}$. Then the composite precursor solution was produced by mixing PVP/AI and $\mathrm{LN} / \mathrm{H}_{2} \mathrm{O}$ solutions. Composite fibers were blow-spun by using the precursor solution $(\mathrm{PVP}+\mathrm{LN}) /\left(\mathrm{AI}+\mathrm{H}_{2} \mathrm{O}\right)$ keeping the $\mathrm{AI} / \mathrm{H}_{2} \mathrm{O}$ ratio at $70: 30(v: v)$, which was determined as the better solvent condition to produce polymeric fibers.

Composite fibers were thermally treated at $600{ }^{\circ} \mathrm{C}$ for $4 \mathrm{~h}$ by using $1{ }^{\circ} \mathrm{C} / \mathrm{min}$ as heating rate. The ceramic phase formation was investigated by X-ray diffraction (XRD, Shimadzu model XRD-6000). Rietveld refinement was employed to study the structural characteristics of the LN fibers. The crystallite size was calculated by using the Scherrer's equation [12]. The fiber morphology was characterized by scanning electron microscopy (SEM, ZEISS model EVO-LS15).

The obtained microfibers/powders were mixed with polyvinyl alcohol (PVA) which acted as a binder to reduce the brittleness of the pellets. The powders were pressed into cylindrical pellets using $\mathrm{KBr}$ hydraulic press at a pressure of $4 \times 10^{6} \mathrm{~N} / \mathrm{m}^{2}$. The pellets were about $12 \mathrm{~mm}$ in diameter and $1-2 \mathrm{~mm}$ in thickness. Then the pellets were sintered at $600{ }^{\circ} \mathrm{C}$. The pellets were polished by fine emery paper to make the surfaces both flat and parallel. To study the electrical properties of the fibers, both flat surfaces of the pellets were electroded with air-drying conducting silver paste. After electroding, the pellets were dried at $150{ }^{\circ} \mathrm{C}$ for $1 \mathrm{~h}$ to remove moisture, if any, and then cooled to room temperature before taking any electrical measurement. The electrical parameters (resistance and capacitance) of the samples were measured by using an LCR meter (HIOKI, Model-3532) in the frequency range of $10^{2}-10^{6} \mathrm{~Hz}$ and the temperature range of $25-450{ }^{\circ} \mathrm{C}$.

\section{Results and discussion}

\section{1 Structural and micro-structural properties}

The phase identification of the sintered LN fibers was performed by XRD. The XRD pattern of the LN fibers 
annealed at $600{ }^{\circ} \mathrm{C}$ for $4 \mathrm{~h}$ shown in Fig. 1 indicates that these fibers are crystallized in pure $\mathrm{LiNbO}_{3}$ phase. The XRD pattern also reveals a non-preferred orientation. According to the literature, usually a randomly oriented polycrystalline $\mathrm{LiNbO}_{3}$ would have a peak intensity ratio of 2.5 between the (012) and (104) $h k l$ peaks. The same ratio for $(012) /(104)$ is observed in our sample. Rietveld refinement was performed by using the ICSD crystallographic data reference 247737 $[12,13]$. The crystallite size was estimated by using Scherrer's equation and found to be around $466 \mathrm{~nm}$.

The influence of deionized water content on PVP fiber formation was initially investigated because the precursor ceramic solution used deionized water as solvent. Since PVP is a hygroscopic polymer, a large amount of water can prejudice the fiber morphology or even prevent their formation. On the other hand, it is highly interesting that the composite solution presents the maximum of ceramic precursor as possible. Figure 2(a) is the SEM image of PVP fibers obtained by using $\mathrm{AI} / \mathrm{H}_{2} \mathrm{O}$ (70:30) as solvent. At this proportion the solution viscosity is found around $430 \mathrm{cP}$ at $26{ }^{\circ} \mathrm{C}$. It shows long and cylindrical fibers with smooth and uniform morphology. Only small defects marked by the junction of the fibers at the contact points are observed. This junction of fibers occurs because of the presence of solvent in the fibrous materials and incomplete evaporation of the solvent (mainly caused by the presence of water and the fast production rate of the fibers which is an intrinsic characteristic of the technique employed). The fiber diameter distribution (Fig. 2(b)) shows a monomodal wide function with maximum centered around $800 \mathrm{~nm}$.

Composite fibers of PVP/LN were deposited at the

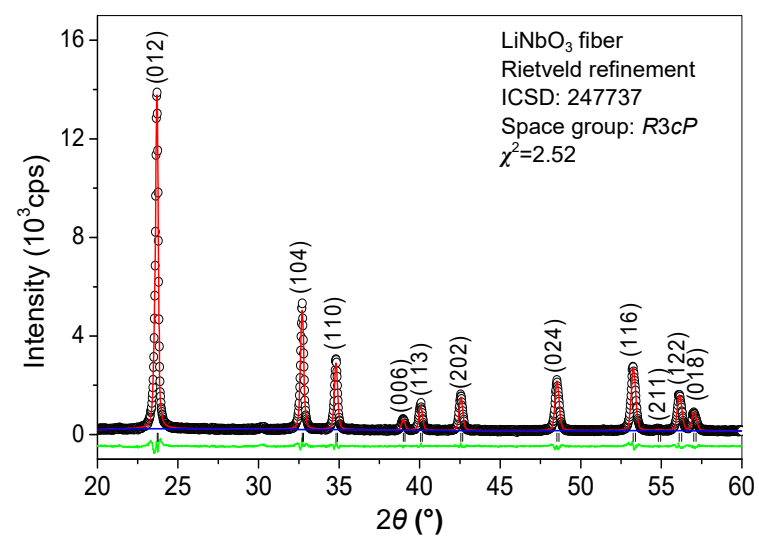

Fig. 1 XRD pattern of $\mathrm{LiNbO}_{3}$ fibers: hole pointsexperimental data, full line- experimental adjust. The $h k l$ values are indicated as per the literature [12]. same previous conditions, i.e., PVP dissolved in $\mathrm{AI} / \mathrm{H}_{2} \mathrm{O}$ (70:30) by replacing the water content with LN solution. After thermal treatment at $600{ }^{\circ} \mathrm{C}$ for $4 \mathrm{~h}$ by using $1{ }^{\circ} \mathrm{C} / \mathrm{min}$ as heating rate, the lithium niobate $\left(\mathrm{LiNbO}_{3}\right)$ ceramic fibers were successfully obtained. Figure 2(c) shows the SEM image of LN fiber formation of the samples after the thermal treatment. The fiber diameter distribution (Fig. 2(d)) shows a monomodal wide function with maximum centered around $800 \mathrm{~nm}$ which is the same as observed for pure PVP. A large amount of defects are identified in the image (Fig. 3) (some of them are attributed to bead formation as shown in Fig. 3(a)) [14]. The bead formation is a usual occurrence in the fiber deposition process related to the surface tension of the solution. This can be improved by the presence of solvent in the resultant composite fiber. Besides, it is also identified the fusion between nearby fibers. The entire drops of precursor solution deposited with the fibers are also identified as shown in Fig. 3(b). The presence of the drops is observed by a semi-spherical crystallized structure formation on the ceramic fibers. Besides these defects, uniform and cylindrical ceramic fibers clearly constituted by ceramic grains, as seen by SEM analysis, are also obtained.

\section{2 Dielectric properties}

Figure 4 shows the frequency dependence of dielectric constant $\left(\varepsilon_{\mathrm{r}}\right)$ and variation of loss tangent $(\tan \delta)$ with frequency at room temperature. It has been observed that both the parameters decrease with increase in frequency which indicates a normal behavior of dielectric materials having mobile charge carriers (i.e., ions and electrons) at room temperature [15]. At low frequency, normally all types of polarizations exist. However, the ionic and electronic polarization exists in the high-frequency range and the other polarizations become affectless [16].

Figure 5 shows the variation of dielectric constant $\left(\varepsilon_{\mathrm{r}}\right)$ as a function of temperature at different frequencies of $\mathrm{LiNbO}_{3}$ fibers. The value of dielectric constant $\left(\varepsilon_{\mathrm{r}}\right)$ increases gradually with increasing temperature. Moreover, an anomaly is observed (at around $355^{\circ} \mathrm{C}$ ) at $500 \mathrm{kHz}$ and $1 \mathrm{MHz}$. The peak value of dielectric constant $\left(\varepsilon_{\max }\right)$ is found to be 73 and 68 at $500 \mathrm{kHz}$ and $1 \mathrm{MHz}$ respectively at $355^{\circ} \mathrm{C}$. Figure 6 shows the variation of loss tangent $(\tan \delta)$ as a function of temperature at different frequencies of 


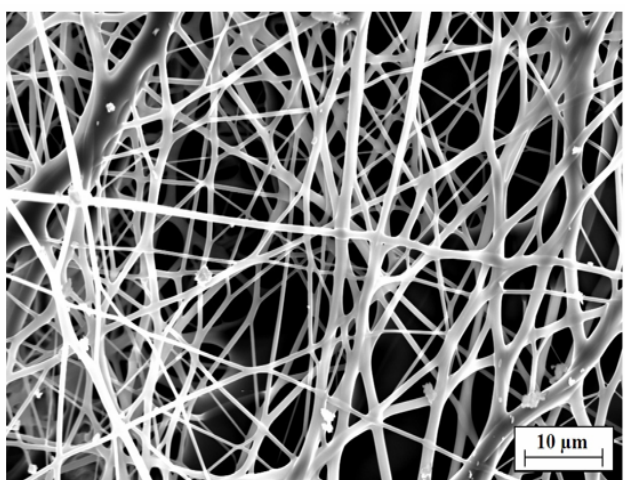

(a)

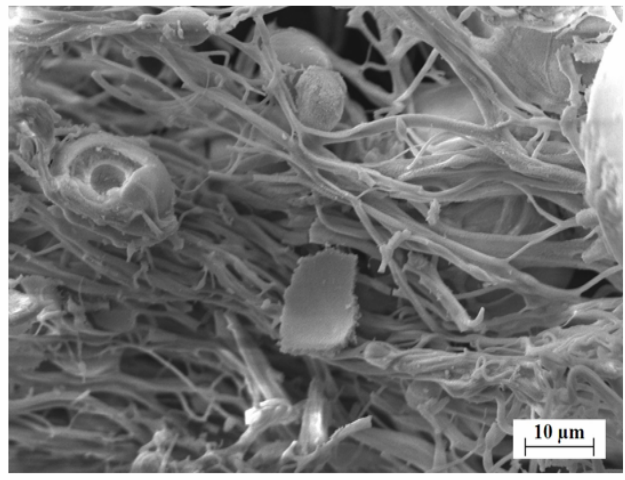

(c)

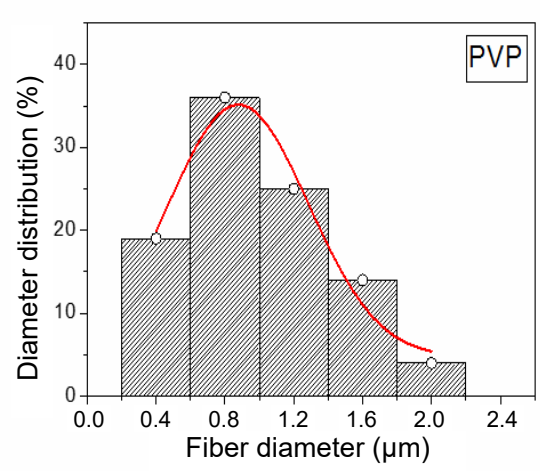

(b)

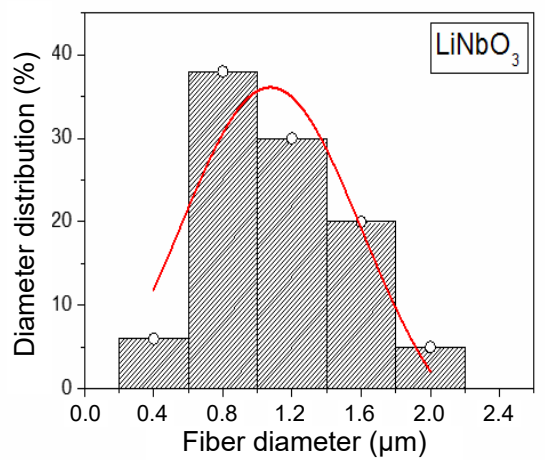

(d)

Fig. 2 (a) SEM image of fibers obtained from pure PVP solution by using $\mathrm{AI} / \mathrm{H}_{2} \mathrm{O}$ (70:30) as solvent. (b) Histogram of PVP fiber diameter distribution. (c) $\mathrm{LiNbO}_{3}$ fibers heat treated at $600{ }^{\circ} \mathrm{C}$ for $4 \mathrm{~h}$. (d) Histogram of $\mathrm{LiNbO}_{3}$ fiber diameter distribution.

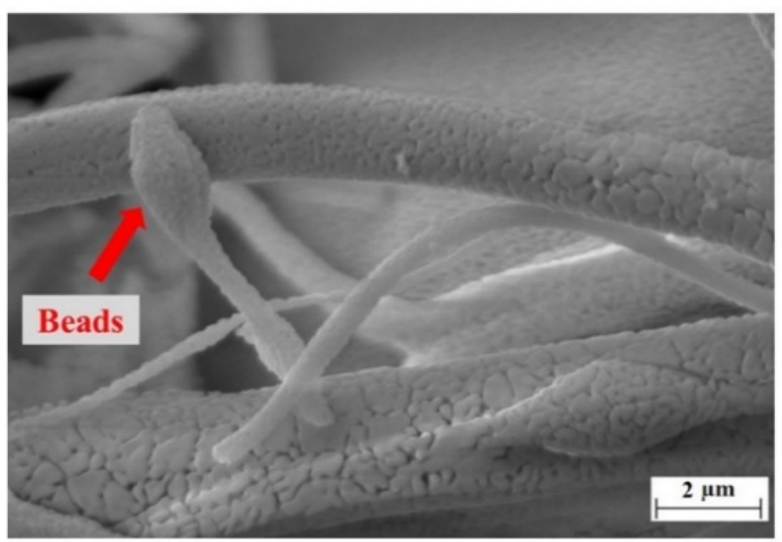

(a)

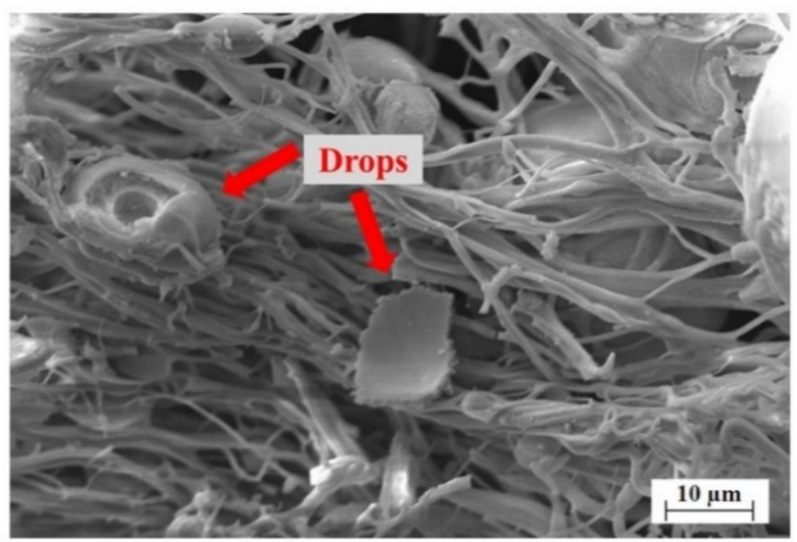

(b)

Fig. 3 SEM images of lithium niobate (LN) fibers with magnification of (a) $18000 \times$ and (b) $3000 \times$.

$\mathrm{LiNbO}_{3}$ fibers. The variation of $\tan \delta$ follows the same behavior as that of $\varepsilon_{\mathrm{r}}$. The value of $\tan \delta$ increases with rise in temperature. This increase in $\tan \delta$ may be due to the enhancement in conductivity [17].

\section{3 Impedance properties}

Complex impedance spectroscopy (CIS) [18] is a unique and powerful technique to characterize the electrical behavior of a system in which a number of strongly coupled processes exist such as grain, grain boundary, and interface properties of ceramics. This also includes the determination of capacitance (bulk and grain boundary), relaxation frequency, and electrical conductivity. Usually, a polycrystalline material gives grain and grain boundary properties 


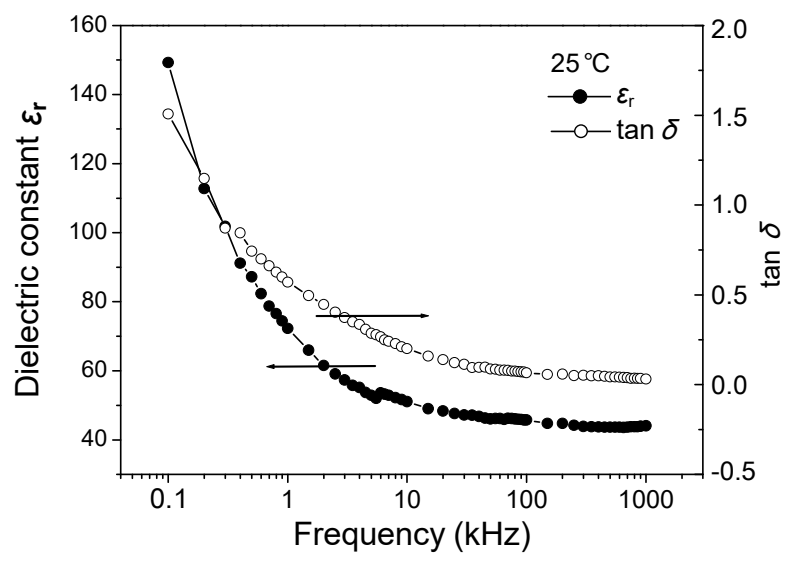

Fig. 4 Frequency dependence of dielectric constant $\left(\varepsilon_{\mathrm{r}}\right)$ and variation of loss tangent $(\tan \delta$ ) with frequency at room temperature of $\mathrm{LiNbO}_{3}$ fibers.

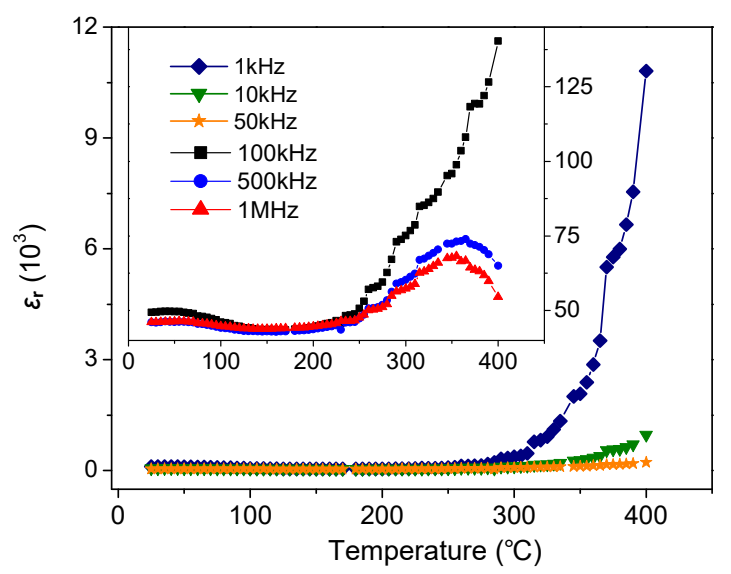

Fig. 5 Variation of dielectric constant $\left(\varepsilon_{\mathrm{r}}\right)$ as a function of temperature at different frequencies of $\mathrm{LiNbO}_{3}$ fibers.

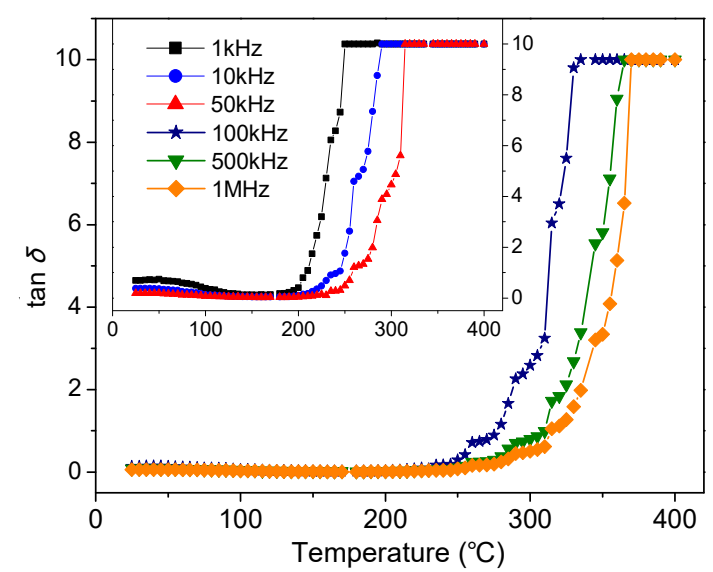

Fig. 6 Variation of loss tangent $(\tan \delta)$ as a function of temperature at different frequencies of $\mathrm{LiNbO}_{3}$ fibers.

with different time constants. Generally, the data in the complex plane is represented in any of the four basic formalisms. These are complex impedance $\left(Z^{*}\right)$, complex admittance $\left(Y^{*}\right)$, complex permittivity $\left(\varepsilon^{*}\right)$, complex electric modulus $\left(M^{*}\right)$, which are related to each other as $Z^{*}=Z^{\prime}-\mathrm{j} Z^{\prime \prime}, M^{*}=M^{\prime}+\mathrm{j} M^{\prime \prime}, Y^{*}=$ $Y^{\prime}+\mathrm{j} Y^{\prime \prime}, \varepsilon^{*}=\varepsilon^{\prime}-\mathrm{j} \varepsilon^{\prime \prime}$, and $\tan \delta=\varepsilon^{\prime \prime} / \varepsilon^{\prime}=M^{\prime \prime} / M^{\prime}=$ $Z^{\prime} / Z^{\prime \prime}$, where $\mathrm{j}=(-1)^{1 / 2}$ is the imaginary factor, and the symbols $\left(Z^{\prime}, M^{\prime}, Y^{\prime}, \varepsilon^{\prime}\right)$ and $\left(Z^{\prime \prime}, M^{\prime \prime}, Y^{\prime \prime}, \varepsilon^{\prime \prime}\right)$ are real and imaginary components of impedance, electrical modulus, admittance, and permittivity respectively. The complex impedance of "electrode/ sample/electrode" configuration can be explained with the series combination of parallel $\mathrm{RC}(\mathrm{R}=$ resistance and $\mathrm{C}=$ capacitance) circuit. Thus, the impedance analysis is unambiguous, and provides a true picture of the electrical behavior of the materials.

Figure 7 shows the complex impedance spectrum ( $Z^{\prime}$ vs. $Z^{\prime \prime}$ ) of $\mathrm{LiNbO}_{3}$ fibers at various temperatures $\left(325-400{ }^{\circ} \mathrm{C}\right)$. Single semicircular arcs have been observed and found to be depressed with their centers below the real axis (not shown) which confirms the existence of non-Debye type relaxation. It is also observed that the intercept point on the real axis shifts towards the origin with the increase in temperature. This indicates the decrease in the resistive property, i.e., so called bulk resistance $\left(R_{\mathrm{b}}\right)$ of the materials like semiconductor [19-21].

Figure 8 shows the variation of $Z^{\prime}$ with frequency of $\mathrm{LiNbO}_{3}$ fibers at different temperatures (325$\left.400{ }^{\circ} \mathrm{C}\right)$. It is observed that the magnitude of $Z^{\prime}$ decreases with rise in temperature in the low-frequency region and appears to merge in the high-frequency region (independent of frequency). This may be due to the release of space charges with rise in temperature



Fig. 7 Complex impedance spectrum $\left(Z^{\prime}\right.$ vs. $\left.Z^{\prime \prime}\right)$ of $\mathrm{LiNbO}_{3}$ fibers at various temperatures $\left(325-400{ }^{\circ} \mathrm{C}\right)$ and variation of $\tau$ as a function of inverse of absolute temperature (inset). 




Fig. 8 Variation of $Z^{\prime}$ with frequency $\left(10^{2}-10^{6} \mathrm{~Hz}\right)$ of $\mathrm{LiNbO}_{3}$ fibers at different temperatures.

$[22,23]$. It can be attributed to the reduction in barrier properties of the materials with rise in temperature and responsible for the enhancement of conductivity $[24,25]$. At a particular frequency, $Z^{\prime}$ becomes independent of frequency.

The loss spectrum ( $Z^{\prime \prime}$ vs. frequency) at different temperatures is shown in Fig. 9. This is broader than the ideal Debye curve and asymmetric. As the temperature increases, the peak position shifts towards the higher-frequency side and exists a strong dispersion of $Z^{\prime \prime}$. The peak width of loss spectrum exhibits the distribution of relaxation time. The relaxation effect and asymmetric spectrum (observed above $325^{\circ} \mathrm{C}$ ) suggest that non-Debye type of relaxation process occurs in this material.

Generally, the impedance data is used to evaluate the relaxation time $(\tau)$ of the electrical phenomena in the materials using the relation $\tau=1 / \omega=1 / 2 \pi f_{\mathrm{r}}$, where

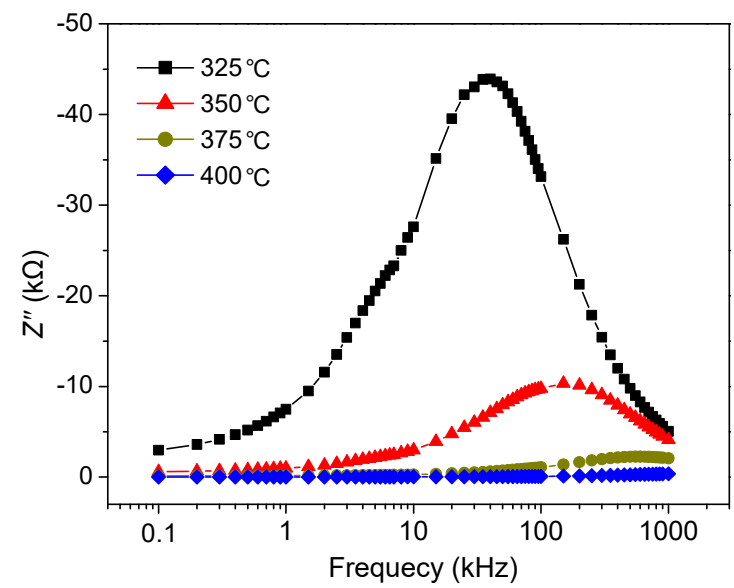

Fig. 9 Variation of $Z^{\prime \prime}$ as a function of frequency at different temperatures of $\mathrm{LiNbO}_{3}$ fibers. $f_{\mathrm{r}}$ is the relaxation frequency. The variation of $\tau$ as a function of inverse of absolute temperature is shown in Fig. 7(inset). It appears to be linear and follows the Arrhenius relation $\tau=\tau_{0} \exp \left(-E_{\mathrm{a}} / k_{\mathrm{B}} T\right)$, where $\tau_{0}$ is the pre-exponential factor, $E_{\mathrm{a}}$ is the activation energy, $k_{\mathrm{B}}$ is the Boltzmann constant, and $T$ is the absolute temperature. The activation energy calculated from impedance plot is found to be $1.5 \mathrm{eV}$.

\section{4 Modulus properties}

The complex modulus spectroscopy is a very important and convenient tool to determine, analyze, and interpret the dynamical aspects of electrical transport process in the materials such as the parameters carrier/ion hopping rate, conductivity relaxation time, etc., where the smallest capacitance occurs in a dielectric system. Modulus spectroscopy plots are particularly useful for separating spectral components of materials having similar resistances but different capacitances. The other advantage of the electric modulus formalism is that the electrode effect is suppressed. The electric modulus $M^{*}(\omega)$ is expressed in the complex modulus formalism $M^{*}(\omega)=$ $\mathrm{j}\left(\omega C_{0}\right) Z^{*}=M^{\prime}+\mathrm{j} M^{\prime \prime}$, where $M^{\prime}=\omega C_{0} Z^{\prime \prime}$ and $M^{\prime \prime}=$ $\omega C_{0} Z^{\prime} \quad[26,27]$

Figure 10 shows the complex modulus spectrum ( $M^{\prime}$ vs. $M^{\prime \prime}$ ) of $\mathrm{LiNbO}_{3}$ fibers at different temperatures. In order to avoid the ambiguity arising out of the presence of grain/grain boundary effect [28], the impedance data is re-plotted in the modulus formalism. Complex impedance spectrum gives more emphasis to elements with larger resistance whereas complex electric modulus with smaller capacitance.

Figure 11 shows the variation of $M^{\prime}$ as a function

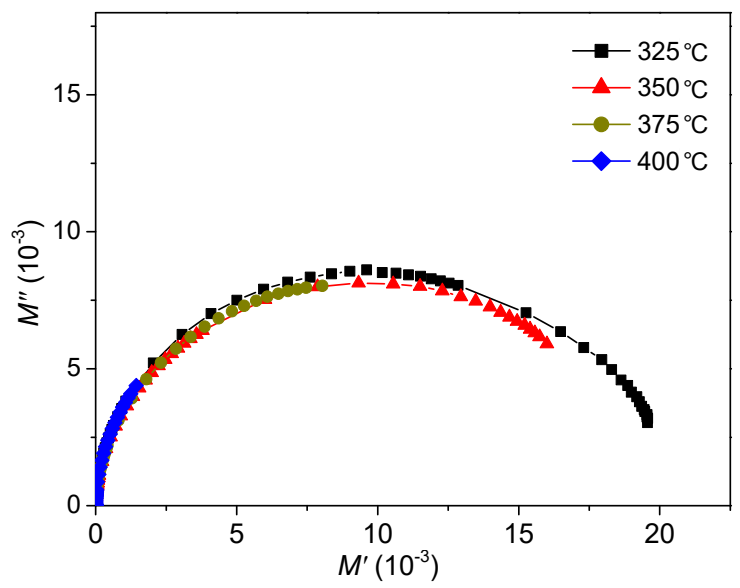

Fig. 10 Variation of $M^{\prime}$ vs. $M^{\prime \prime}$ of $\mathrm{LiNbO}_{3}$ fibers at selected temperatures. 


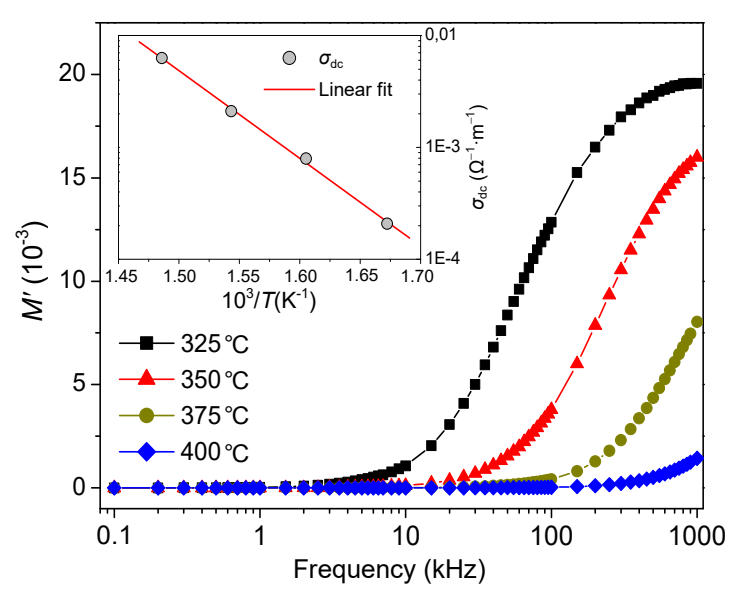

Fig. 11 Variation of $M^{\prime}$ with frequency at different temperatures and variation of dc conductivity with inverse of absolute temperature (inset) of $\mathrm{LiNbO}_{3}$ fibers.

of frequency for $\mathrm{LiNbO}_{3}$ fibers at selected temperatures $\left(325-400{ }^{\circ} \mathrm{C}\right)$. At lower frequencies, $M^{\prime}$ tends to be very small (approximately zero). There is a continuous dispersion with increase in frequency. This may be due to the short-range mobility of charge carriers [29] which confirms the very small contribution of electrode effect. In this figure, $M^{\prime}$ reaches a constant value $\left(M_{\infty}\right)$ at high frequencies for all the temperatures and shows relaxation process occurred in the material.

The variation of $M^{\prime \prime}$ with frequency at different temperatures is shown in Fig. 12. The maxima, $M_{\max }^{\prime \prime}$, shifts towards higher-frequency side with rise in temperature, which shows the correlation between motion of mobile ions [30]. This suggests that the dielectric relaxation is a thermally activated process of

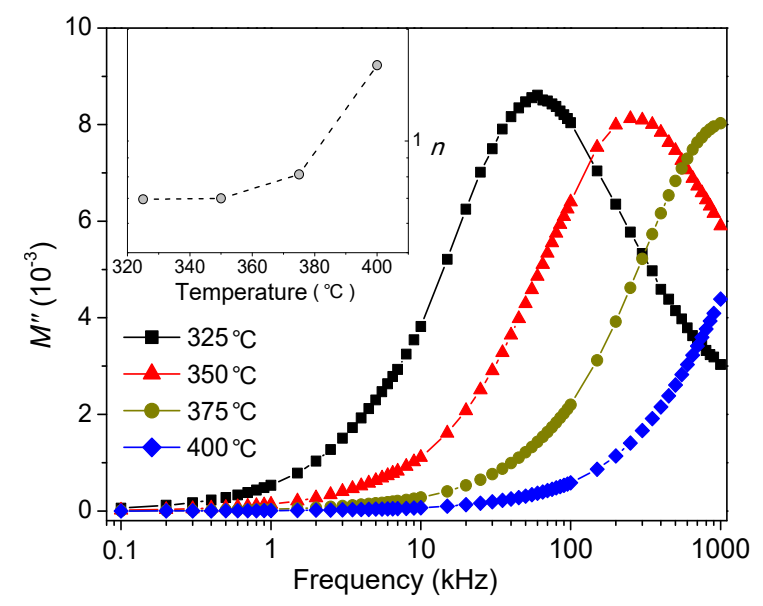

Fig. 12 Variation of $M^{\prime \prime}$ with frequency at different temperatures and variation of $n$ with temperature (inset) of $\mathrm{LiNbO}_{3}$ fibers. the material. This type of effect has also been reported in the modulus spectrum of some ionic conductors [31]. The asymmetric peak broadening indicates the spread of relaxation time with different time constant, and hence relaxation is of non-Debye type. The nature of modulus spectrum suggests the existence of hopping mechanism of electrical conduction in the material [29].

\section{5 Electrical conductivity}

The frequency dependent conductivity spectrum of $\mathrm{LiNbO}_{3}$ is shown in Fig. 13 at selected temperatures $\left(325-400{ }^{\circ} \mathrm{C}\right)$. This spectrum displays a low-frequency plateau region and a high-frequency dispersive region. The plateau region corresponds to the frequency independent part of dc conductivity. Furthermore, the increase of a unique value of conductivity with rise in temperature indicates that the electrical conduction in the material is a thermally activated process. The nature of the conductivity in solids is generally analyzed using Jonscher's power law $\sigma_{\mathrm{ac}}(\omega)=\sigma(0)+$ $A \omega^{n}$ [32], where $\sigma(0)$ is the frequency independent (electronic or dc) part of ac conductivity, $n$ is the frequency exponent in the range of $0 \leqslant n \leqslant 1, \omega$ is the angular frequency of applied ac field, and $A$ is the temperature dependent parameter. According to Funke [33], the values of $n$ have a physical meaning, i.e., $n \leqslant 1$ means that the hopping motion involves a translational motion with a sudden hopping, whereas $n>1$ means the motion involves localized hopping without the species leaving the neighborhood. It is also

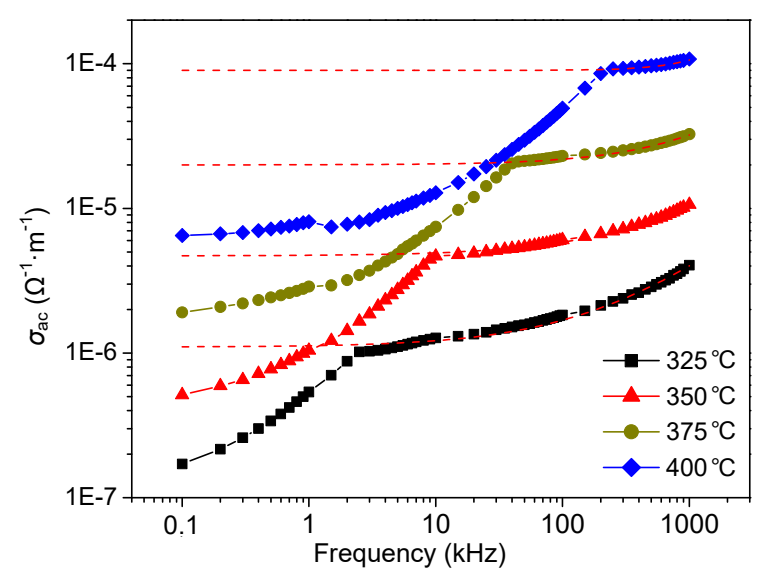

Fig. 13 Variation of ac conductivity $\left(\sigma_{\mathrm{ac}}\right)$ with frequency of $\mathrm{LiNbO}_{3}$ fibers at different temperatures. 
reported that the exponent $n$ is not limited to values below 1 [34]. Due to the localization of charge carriers, formation of polarons takes place and the hopping conduction occurs between the nearest neighbouring sites. This suggests that the electrical conduction in $\mathrm{LiNbO}_{3}$ is a thermally activated process. The non linear curve fit to Jonscher's power law for the sample at different temperatures $\left(325-400{ }^{\circ} \mathrm{C}\right)$ is shown in Fig. 13. The fitting parameters $A, n$, and $\sigma(0)$ are calculated and given in Table 1 . The variation of $n$ with temperature is shown in Fig. 12(inset). It is observed that the value of $n$ increases linearly with increase in temperature. This may be a result of the rise of the electrode polarization contribution with temperature [35]. This type of behavior may be associated with the small polaron (SP) tunnelling models.

The variation of $\sigma_{\mathrm{dc}}$ (bulk) with inverse of absolute temperature $\left(10^{3} / T\right)$ is shown in Fig. 11(inset). The bulk conductivity of the material is evaluated from the complex impedance plots of the sample at selected temperatures using the relation $\rho=l / R_{\mathrm{b}} A$, where $l$ is the thickness, $R_{\mathrm{b}}$ is the bulk resistance, and $A$ is the area of cross section of the sample. The nature of variation of the material follows the Arrhenius relation $\sigma_{\mathrm{dc}}=\sigma_{0} \exp \left(-E_{\mathrm{a}} / k_{\mathrm{B}} T\right)$, where $\sigma_{0}$ is the pre-exponential factor. It is observed that the $\mathrm{dc}$ conductivity increases (i.e., bulk resistivity decrease) with rise in temperature and shows negative temperature coefficient of resistance (NTCR) behaviour like semiconductor. The temperature dependence of $\mathrm{dc}$ conductivity indicates that the electrical conduction in the material is a thermally activated process. The value of activation energy $\left(E_{\mathrm{a}}\right)$ of the material is found to be $1.5 \mathrm{eV}$ in the high-temperature region $\left(325-400{ }^{\circ} \mathrm{C}\right)$. This small amount of energy is required for the conduction process in the material and may be due to the singly ionized oxygen vacancies at higher temperature.

\begin{tabular}{lccc}
$\begin{array}{l}\text { Table } 1 \\
\text { Fitting parameters of } \mathrm{LiNbO}_{3} \text { fibers obtained } \\
\text { from the Jonscher's } \\
\text { temperatures }\end{array}$ & power law at & different \\
\hline$T\left({ }^{\circ} \mathrm{C}\right)$ & $\sigma_{\mathrm{dc}}\left(\Omega^{-1} \cdot \mathrm{m}^{-1}\right)$ & $A$ & $n$ \\
\hline 325 & 0.0011 & $1.9378 \times 10^{-7}$ & 0.69627 \\
350 & 0.0047 & $3.6143 \times 10^{-7}$ & 0.7 \\
375 & 0.02 & $1.6272 \times 10^{-7}$ & 0.81235 \\
400 & 0.09 & $4.0422 \times 10^{-12}$ & 1.6 \\
\hline
\end{tabular}

\section{Conclusions}

The LN ceramic fibers were prepared from a simple precursor solution using the "blow-spinning" technique. The SEM images of ceramic fibers exhibited a diameter range from 0.4 to $2 \mu \mathrm{m}$. Few defects in the fiber morphology and cylindrical fibers with well-defined morphology were observed with homogeneous smooth surfaces. The solution and deposition parameters were refined to produce defect free fibers. Impedance spectroscopy technique was used to characterize the electrical properties of the material. Modulus analysis confirmed the presence of hopping mechanism in the material. The electrical conduction of the sample was due to the contribution of bulk and grain boundary effects. The bulk resistance decreased with rise in temperature which indicates a typical NTCR behavior of the material. The ac conductivity spectrum was found to obey Jonscher's universal power law whereas dc conductivity showed a typical Arrhenius type of electrical conductivity. The activation energy of the material was found to be $1.5 \mathrm{eV}$.

\section{References}

[1] Dai Y, Liu W, Formo E, et al. Ceramic nanofibers fabricated by electrospinning and their applications in catalysis, environmental science, and energy technology. Polym Adv Technol 2011, 22: 326-338.

[2] Sigmund W, Yuh J, Park H, et al. Processing and structure relationships electrospinning of ceramic fiber systems. J Am Ceram Soc 2006, 89: 395-407.

[3] Medeiros ES, Glenn GM, Klamczynski AP, et al. Solution blow spinning: A new method to produce micro- and nanofibers from polymer solutions. J Appl Polym Sci 2009, 113: 2322-2330.

[4] Bhardwaj N, Kundu SC. Electrospinning: A fascinating fiber fabrication technique. Biotechnol $A d v$ 2010, 28: 325-347.

[5] Oliveira JE, Moraes EA, Costa RGF, et al. Nano and submicrometric fibers of poly(D,L-lactide) obtained by solution blow spinning: Process and solution variables. J Appl Polym Sci 2011, 122: 3396-3405.

[6] Yuh J, Nino JC, Sigmund WM. Synthesis of barium titanate $\left(\mathrm{BaTiO}_{3}\right)$ nanofibers via electrospinning. Mater Lett 2005, 59: 3645-3647.

[7] Alkoy EM, Dagdeviren C, Papila M. Processing conditions and aging effect on the morphology of PZT electrospun nanofibers, and dielectric properties of the resulting 3-3 PZT/polymer composite. J Am Ceram Soc 2009, 92: 2566-2570.

[8] Maensiri S, Nuansing W, Klinkaewnarong J, et al. 
Nanofibers of barium strontium titanate (BST) by sol-gel processing and electrospinning. $J$ Colloid Interface Sci 2006, 297: 578-583.

[9] Li D, Xia Y. Fabrication of titania nanofibers by electrospinning. Nano Lett 2003, 3: 555-560.

[10] Bartasyte A, Plausinaitiene V, Abrutis A, et al. Identification of $\mathrm{LiNbO}_{3}, \mathrm{LiNb}_{3} \mathrm{O}_{8}$ and $\mathrm{Li}_{3} \mathrm{NbO}_{4}$ phases in thin films synthesized with different deposition techniques by means of XRD and Raman spectroscopy. J Phys Condens Matter 2013, 25: 205901.

[11] Díaz-Moreno C, Farias R, Hurtado-Macias A, et al. Multiferroic response of nanocrystalline lithium niobate. J Appl Phys 2012, 111: 07D907.

[12] Azaroff LV, Buerguer MJ. The Powder Method in X-ray Crystallography. New York: McGran-Hill, 1958.

[13] Jeong I-K, Park S. Correlated thermal motion in ferroelectric $\mathrm{LiNbO}_{3}$ studied using neutron total scattering and a Rietveld analysis. J Korean Phys Soc 2011, 59: 2756.

[14] Fong H, Chun I, Reneker DH. Beaded nanofibers formed during electrospinning. Polymer 1999, 40: 4585-4592.

[15] Anderson JC. Dielectrics. London: Chapman \& Hall, 1964.

[16] Lidorenko NS, Zil'bervarg VE, Nagaev EL. Dielectric constants of solid eletrolytes and transition to superionic state. Sov Phys JETP 1980, 51: 89-93.

[17] Khatri P, Behera B, Srinivas V, et al. Structural and dielectric properties of $\mathrm{Ba}_{3} \mathrm{~V}_{2} \mathrm{O}_{8}$ ceramics. Curr Appl Phys 2009, 9: 515-519.

[18] Macdonald JR. Impedance Spectroscopy. New York: Wiley, 1987.

[19] Jawahar K, Behera B, Choudhary RNP. Dielectric and impedance properties of $\mathrm{Nd}_{3 / 2} \mathrm{Bi}_{3 / 2} \mathrm{Fe}_{5} \mathrm{O}_{12}$ ceramics. J Mater Sci: Mater Electron 2009, 20: 872-878.

[20] Sinclair DC, West AR. Impedance and modulus spectroscopy of semiconducting $\mathrm{BaTiO}_{3}$ showing positive temperature coefficient of resistance. J Appl Phys 1989, 66: 3850.

[21] Satpathy S, Mohanty N, Behera A, et al. Dielectric and electrical properties of $0.5\left(\mathrm{BiGd}_{0.05} \mathrm{Fe}_{0.95} \mathrm{O}_{3}\right)-0.5\left(\mathrm{PbZrO}_{3}\right)$ composite. Mater Sci-Poland 2014, 32: 59-65.

[22] Behera AK, Mohanty NK, Satpathy SK, et al. Investigation of complex impedance and modulus properties of $\mathrm{Nd}$ doped $0.5 \mathrm{BiFeO}_{3}-0.5 \mathrm{PbTiO}_{3}$ multiferroic composites. Cent Eur J Phys 2014, 12: 851-861.

[23] Płcharski J, Weiczorek W. PEO based composite solid electrolyte containing nasicon. Solid State Ionics 1988,
28-30: 979-982.

[24] Provenzano V, Boesch LP, Volterra V, et al. Electrical relaxation in $\mathrm{Na}_{2} \mathrm{O} \cdot 3 \mathrm{SiO}_{2}$ glass. $J$ Am Ceram Soc 1972, 55: 492-496.

[25] Jain H, Hsieh CH. 'Window' effect in the analysis of frequency dependence of ionic conductivity. J Non-Cryst Solids 1994, 172-174: 1408-1412.

[26] Sen S, Pramanik P, Choudhary RNP. Impedance spectroscopy study of the nanocrystalline ferroelectric (PbMg)(ZrTi)O 3 system. Appl Phys A 2006, 82: 549-557.

[27] Réau J-M, Simon A, Omari ME, et al. Impedance spectroscopy analysis of $\mathrm{Pb}_{5} \mathrm{~A}_{13} \mathrm{~F}_{19}$. J Eur Ceram Soc 1999, 19: 777-779.

[28] Choudhary RNP, Pradhan DK, Tirado CM, et al. Impedance characteristics of $\mathrm{Pb}\left(\mathrm{Fe}_{2 / 3} \mathrm{~W}_{1 / 3}\right) \mathrm{O}_{3}-\mathrm{BiFeO}_{3}$ composites. Phys Status Solidi b 2007, 244: 2254-2266.

[29] Behera B, Nayak P, Choudhary RNP. Study of complex impedance spectroscopic properties of $\mathrm{LiBa}_{2} \mathrm{Nb}_{5} \mathrm{O}_{15}$ ceramics. Mater Chem Phys 2007, 106: 193-197.

[30] Borsa F, Torgeson DR, Martin SW, et al. Relaxation and fluctuations in glassy fast-ion conductors: Wide-frequencyrange NMR and conductivity measurements. Phys Rev $B$ 1992, 46:795-800.

[31] Elliott SR. Use of the modulus formalism in the analysis of ac conductivity data for ionic glasses. J Non-Cryst Solids 1994, 170: 97-100.

[32] Jonscher AK. The 'universal' dielectric response. Nature 1977, 267: 673-679.

[33] Funke K. Jump relaxation in solid electrolytes. Prog Solid State Ch 1993, 22: 111-195.

[34] Sakellis I, Papathanassiou AN, Grammatikakis J. Transformation of polarons to bipolarons in disordered matter. Appl Phys Lett 2008, 92: 222108.

[35] Mahamoud H, Louati B, Hlel F, et al. Conductivity and dielectric studies on $\left(\mathrm{Na}_{0.4} \mathrm{Ag}_{0.6}\right)_{2} \mathrm{PbP}_{2} \mathrm{O}_{7}$ compound. Bull Mater Sci 2011, 34: 1069-1075.

Open Access The articles published in this journal are distributed under the terms of the Creative Commons Attribution 4.0 International License (http://creativecommons. org/licenses/by/4.0/), which permits unrestricted use, distribution, and reproduction in any medium, provided you give appropriate credit to the original author(s) and the source, provide a link to the Creative Commons license, and indicate if changes were made. 